Original Research Article

\title{
Use of NIRS for the assessment of meat quality traits in open-air free-range Iberian pigs
}

\author{
Miguel Ángel Fernández-Barroso ${ }^{\mathrm{a}, \mathrm{b}, *}$, Silvia Parrini ${ }^{\mathrm{c}}$, María Muñoz ${ }^{\mathrm{a}, \mathrm{b}}$, \\ Patricia Palma-Granados ${ }^{\mathrm{a}, \mathrm{b}}$, Gema Matos ${ }^{\mathrm{d}}$, Luisa Ramírez ${ }^{\mathrm{d}}$, Alessandro Crovetti ${ }^{\mathrm{c}}$, \\ Juan María García-Casco $^{\text {a,b }}$, Riccardo Bozzi ${ }^{c}$ \\ ${ }^{a}$ Centro Nacional de I+D del Cerdo Ibérico, INIA, Zafra, 06300, Spain \\ ${ }^{\mathrm{b}}$ INIA, Departamento de Mejora Genética Animal, Madrid, 28040, Spain \\ ${ }^{\mathrm{c}}$ Dipartimento di Scienze e Tecnologie Agrarie, Alimentari, Ambientali e Forestali (DAGRI), Università di Firenze, Italy \\ d Sánchez Romero Carvajal—Jabugo, SRC, Huelva, 21290, Spain
}

\section{A R T I C L E I N F O}

\section{Keywords:}

NIRS

Iberian pig

Meat quality

Intact and minced samples

\begin{abstract}
A B S T R A C T
Near infrared spectroscopy (NIRS) can be useful in order to determine meat quality traits as a rapid and nondestructive technique. The aim of the present study is to assess the accuracy of NIRS technique to determine meat quality traits on Longissimus thoracis et lumborum muscle of open-air free-range Iberian pig ( $\mathrm{n}=287$ ) both in intact and minced samples. Traits were measured by instrumental-chemical techniques: colour $\left(\mathrm{L}^{*}, \mathrm{a}^{*}\right.$ and $\left.\mathrm{b}^{*}\right)$, myoglobin content, centrifuge force water loss and texture: shear force and texture profile analysis (TPA: hardness, cohesiveness, springiness, chewiness). Calibration models between instrumental-chemical measures and NIRS spectral data were developed employing partial least square regressions (PLS). The samples were split in two random datasets ( $80 \%$ in training set, $20 \%$ in external validation set). An internal full cross-validation method was applied. Results were evaluated in terms of coefficient of determination $\left(R^{2}\right)$, root-mean-square error (RMSE), residual prediction deviation (RPD) and range error ratio (RER). Full spectral range was used to develop mathematical equations. As regard external validation procedure, the highest coefficients of determination $\left(R^{2} p\right)$ in intact loin samples were achieved for hardness, redness $\left(a^{*}\right)$ and yellowness $\left(b^{*}\right)\left(0.7<R^{2} p<0.8\right)$, while for minced samples lightness $\left(L^{*}\right)$, myoglobin content and texture parameters obtained always $R^{2} p>0.7$. The models developed suggest the ability of NIRS for quantitative prediction of shear force and for a rough classification of colour parameters $\mathrm{L}^{*}, \mathrm{a}^{*}, \mathrm{~b}^{*}$ in minced samples as well as hardness in intact samples.
\end{abstract}

\section{Introduction}

The traditional Iberian pig production system is characterized by an open-air, free-range fattening period named Montanera based on ad libitum intake of grass and acorns developed in the Dehesa ecosystem (López-Bote, 1998). The high organoleptic meat quality is influenced by the interaction between this rearing system and the genetic characteristics of the breed. Iberian pigs have a slower growth rate, higher potential of fat deposition and higher proportion of monounsaturated fatty acids with respect to other breeds.

Therefore, high meat quality, which is increasingly demanded by consumers, is one of the main aims of the pork processing industry. Usually, meat characteristics are determined by classical chemical, technological and sensorial analyses. The implementation of these techniques is often expensive, invasive and time consuming; consequently, those methodologies are not adapted for real-time analysis and sometimes generate hazardous waste (Tejerina et al., 2018). This is particularly relevant when meat quality traits are included in breeding programs since a large amount of data are needed, requiring cheaper and less laborious techniques.

Near infrared spectroscopy (NIRS), based on the absorption of electromagnetic radiation at wavelengths in the $780-2500 \mathrm{~nm}$ range, is a recognized methodology for some chemical analyses both in food and feed. Near infrared spectra of foods include broad bands arising from overlapping absorption profiles corresponding mainly to overtones and combinations of vibrational modes involving $\mathrm{C}-\mathrm{H}, \mathrm{OH}$ and $\mathrm{NH}-$ chemical bonds (Osborne, 2000). Nevertheless, this technique requires calibration against a reference method for each parameter of interest

\footnotetext{
* Corresponding author at: Centro de I+D en Cerdo Ibérico, INIA, Zafra, Spain.

E-mail address: fernandez.miguel.inia@gmail.com (M.Á. Fernández-Barroso).
} 
and it requires a multivariate analysis able to associate the spectral absorption of the near infrared region with laboratory data. A large database with high levels of variability representing all possible characteristics of the samples is essential to the development of robust and accurate NIRS predictions (Parrini et al., 2017). NIRS could be useful to replace traditional methods of analysis. In some studies, it has been applied for rapid and non-destructive determination of meat quality traits. Prieto et al. (2009) reported that the NIRS technique has a high potential to predict chemical content of meat and to categorise samples based on their meat quality characteristics. Also, Monteiro-Balage et al. (2015) described the potential of NIRS to correctly classify pork samples into two categories (tough and tender), when they used the visible spectrum combined with a part of the near infrared spectrum. In addition, Prieto et al. (2015) showed the capacity of NIRS to discriminate between pork quality of Lacombe, Duroc and Iberian loins applying the technology directly in processing plants. Nevertheless, apart from its discriminatory ability, the same authors (Monteiro-Balage et al., 2015; Prieto et al., 2009) reported low NIRS performance to accurately estimate quality traits, due to different factors including the type of instrument and spectra regions, the statistical methods adopted, the precision of reference methods as well as the number and type of meat samples that, in the case of fresh meat, are linked to the heterogeneity of intact samples and high water content. The use of Fourier Transform in NIRS (FT-NIRS), which includes improvements in signal-to-noise ratio, spectral resolution and wavelength accuracy, could be a key factor to allow qualitative analyses of the traits, improving measurement precision, time of analysis, and costs.

Several studies have used NIRS as a rapid method applicable to Iberian pig products (Cáceres-Nevado et al., 2019; Pérez-Marín et al., 2009; Solís et al., 2001; Tejerina et al., 2018; Zamora-Rojas et al., 2012, 2013). Overall, these NIRS studies were focused on determining chemical composition, intramuscular fat content and fatty acid profiles, and just few compared intact and minced samples (Cáceres-Nevado et al., 2019; Ortiz et al., 2020; Pérez-Marín et al., 2009). The possibility of determining meat quality traits directly on the intact piece could allow both to avoid meat destruction and to reduce the time required for the analysis. Therefore, it is important to investigate these aspects further.

The aim of the present study was to assess the accuracy of the NIRS technique to determine several meat quality traits in intact and minced samples of loins belonging to Iberian pigs fattened in an open-air freerange system using the full near infrared region. As far as we know, this is the first time that the feasibility of NIRS technique is evaluated in this porcine breed and production system, as a quick and clean technique to simultaneously measure this set of meat quality traits in just one run, which includes water holding capacity, myoglobin content, instrumental colour and texture parameters.

Due to the genetic uniqueness of Iberian pig, associated with lack of introgression of Asian and other lean European breeds alleles (Alves et al., 2003; Ollivier, 2009; SanCristobal et al., 2006), the results found in studies with other pig breeds may not be extrapolated to the Iberian. This simultaneous measurement would be very important for the Iberian pig meat industry and particularly in breeding programs including meat quality parameters as a selection goal, since a large number of records are required.

\section{Materials and methods}

\subsection{Animal material}

Pigs included in the current study were castrated males belonging to a commercial population of an Iberian purebred herd that has been closed for approximately 15 years, as described in Fernández-Barroso et al. (2020). The animals were fed under a restricted feeding regime up to approximately $100 \mathrm{~kg}$ of body weight and managed in semi-extensive system. Then, they were fattened in an open-air free-range system based on ad libitum intake of acorns and grass. A total of 287 animals were slaughtered in the same commercial slaughterhouse in four different slaughter batches in the same year, at an approximate age of 17 months and at an average slaughter weight of $173 \mathrm{~kg}(\mathrm{SD}=13.7)$. Animal handling was carried out according to the regulations of the Spanish Policy for Animal Protection RD 53/2013, which meets the European Union Directive 2010/63/EU about the protection of animals used in research. Research protocols were assessed and approved by the INIA Committee of Ethics in Animal Research, which is the named Institutional Animal Care and Use Committee (IACUC) for the INIA.

\subsection{Sample acquisition, texture, colour parameters and chemical determinations}

Samples of Longissimus thoracis et lumborum (LTL) muscle from 287 pigs were removed from the carcass and both intermuscular fat and connective tissue were separated. The central LTL section was subdivided in two parts (Fig. 1). One part was used for myoglobin content (MB) and centrifuge force loss (CFL) determinations, and the other part was used for determination of texture: shear force, texture profile analysis (TPA: hardness, cohesiveness, springiness, chewiness), instrumental colour parameters (lightness $\mathrm{L}^{*}$, redness $\mathrm{a}^{*}$, and yellowness $\mathrm{b}^{*}$ ), and for NIRS determination. A quality control method removing the outliers was carried out.

All samples were refrigerated and vacuum-packed individually in nylon/polyethylene bags. After packing, the meat portions corresponding to $\mathrm{MB}$ and CFL were introduced in liquid $\mathrm{N}_{2}$ for approximately $20 \mathrm{~s}$ before being stored at $-20{ }^{\circ} \mathrm{C}$ to quickly freeze them to reduce potential water loss and prevent changes in the chemical state of myoglobin. The remaining samples were stored directly at $-20^{\circ} \mathrm{C}$. Prior the analyses, samples were defrosted and tempered until determinations (3-5 ${ }^{\circ} \mathrm{C}$ for $24 \mathrm{~h}$ ). Myoglobin content, CFL, colour parameters and shear force determinations has been previously described in detail by Fernandez-Barroso et al. (2020).

Myoglobin content was determined following Hornsey (1956) and Alberti et al. (2005) with modifications. Absorbance was converted to $\mathrm{mg}$ myoglobin/g muscle following the next equation:

\section{$\frac{\boldsymbol{V} \boldsymbol{f} * \boldsymbol{P M} * 1 \mathbf{k g}}{\boldsymbol{\varepsilon} * \boldsymbol{P s a m p l e} * 1000} * \boldsymbol{D O}$}

Where $\boldsymbol{V} \boldsymbol{f}$ is the final volume of determination $(12.5 \mathrm{ml}), \mathbf{P M}$ is molecular weight of myoglobin, $\varepsilon$ is a coefficient applied (9.52), Psample is sample weight (2.5 g), DO is the optical density absorbance. The method described by Tejerina et al. (2012) was used to measure CFL. Measures of MB and CFL were done in duplicate and then averaged.

A steak of $2 \mathrm{~cm}$ thick was cut from the LTL portion for determination of the colour parameters $\mathrm{L}^{*}, \mathrm{a}^{*}$, and $\mathrm{b}^{*}$ using a colorimeter (CR-400, Konica Minolta, New Jersey, USA) with $8 \mathrm{~mm}$ measuring cell aperture, CIE standard illuminant D65, and $2^{\circ}$ observer. The analysis was performed at a room temperature of approximately $20-21^{\circ} \mathrm{C}$. Each colour measurement was carried out in triplicate and then averaged.

Texture analyses were performed using a texture analyser (Z2.5 apparatus, Zwick Roell, Ulm, Germany). Shear force was measured by Warner-Bratzler test (Honikel, 1998). Eight $2.5 \mathrm{~cm}$ diameter cylinders per sample were prepared. Each cylinder was cut perpendicular to the muscle fibre direction with a Warner-Bratzler blade (HDP/BSW) at a crosshead speed of $1 \mathrm{~mm} / \mathrm{s}$ and the eight measures were averaged ( $\mathrm{kg}$ $\mathrm{cm}^{2}$ ). Regarding the texture profile analysis (TPA), a total of eight cubes of $1 \mathrm{~cm}^{3}$ per sample were prepared. Each cube was compressed twice to $50 \%$ of their original height with a $10 \mathrm{~cm}$ diameter compression plate (time $=0 \mathrm{~s}$ between the two compression cycles), at a crosshead speed of $1 \mathrm{~mm} / \mathrm{s}$ and perpendicularly to the muscle fibre direction. Force-time curves were recorded and the subsequent rheological parameters were calculated (Bourne, 1978): hardness, cohesiveness, springiness and chewiness. Hardness $(\mathrm{N})$ is defined as the maximum peak force during the first compression cycle, cohesiveness as the ratio between the area of 


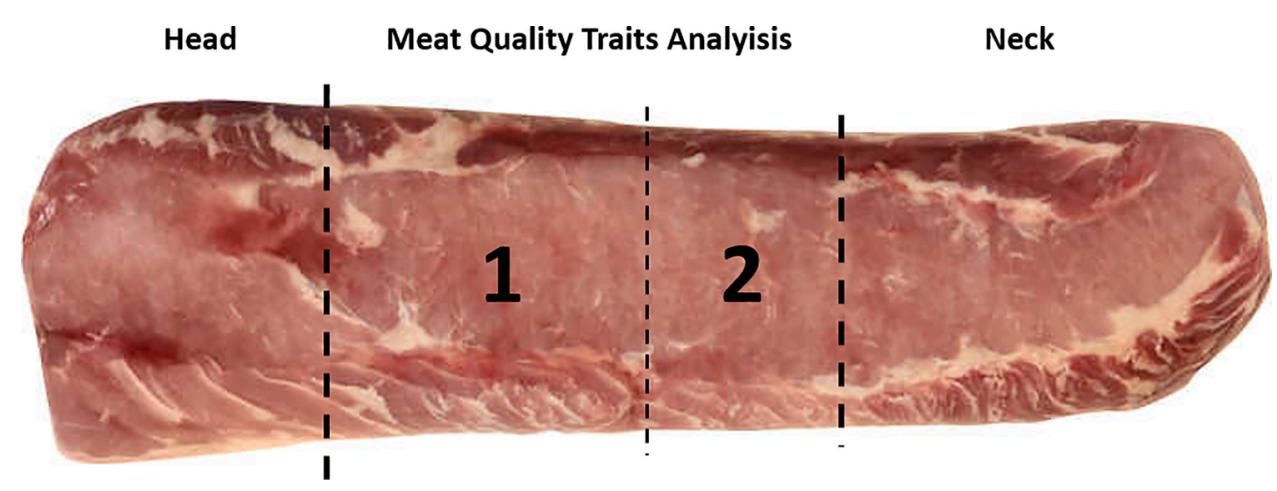

Fig. 1. Assignment of Longissimus thoracis et lumborum muscle for meat quality traits. 1: Texture analysis (Warner-Bratzler shear force (SFF) and texture profile analysis (TPA)), instrumental colour parameters (lightness $\mathrm{L}^{*}$, redness $\mathrm{a}^{*}$, and yellowness $\mathrm{b}^{*}$ ) and NIRS. 2: myoglobin content (MB) and centrifuge force loss (CFL). (For interpretation of the references to colour in this figure legend, the reader is referred to the web version of this article).

the positive force during the second and the first compression cycle, springiness ( $\mathrm{mm}$ ) as the height to which the sample recovers during the time elapsed between the end of the first and the start of the second compression, and chewiness $(\mathrm{N} \times \mathrm{mm})$ as the work needed to chew a solid sample to a steady state of swallowing (hardness $\mathrm{x}$ cohesiveness $\mathrm{x}$ springiness).

\subsection{FT-NIRS data acquisition}

The same samples of approximately $2 \mathrm{~cm}$ thick (around $20 \mathrm{~g}$ ) used for colour parameter measurements were scanned with a FT-NIRS device (Antaris ${ }^{\mathrm{TM}}$ II, Thermo Fisher Scientific, Waltham, Massachusetts, USA) at a lab room temperature of approximately $20-21{ }^{\circ} \mathrm{C}$. Spectral data were collected in reflectance mode and recorded as absorbance $(\log 1 / \mathrm{R}$, where $\mathrm{R}$ is the reflectance). Two-sample presentation modes were evaluated: intact and minced. Two spectra per sample, one for each side, were scanned for the intact mode using a cylindrical cup spinner with a quartz window. For the minced mode, samples were chopped in a homogeniser and two spectra were scanned for each sample. Each spectral measurement was obtained from 32 scans performed at a wave number resolution of $4 \mathrm{~cm}^{-1}$ over the range of 9999 to $4000 \mathrm{~cm}^{-1}$ $(1000-2500 \mathrm{~nm})$ and corrected against the background spectrum of room environment. Average values of the two different replications for each sample were obtained through the OMNIC ${ }^{\text {TM }}$ Software (Thermo Fisher Scientific, Waltham, Massachusetts, USA) both for intact and minced samples.

\subsection{FT-NIRS data pre-treatment}

NIRS pre-processed spectral data were combined with the results of chemical and technological analysis: $\mathrm{MB}, \mathrm{CFL}$, instrumental colour ( $\mathrm{L}^{*}$, $\left.a^{*}, b^{*}\right)$, shear force and TPA (hardness, cohesiveness, springiness and chewiness).

For each trait, an individual calibration model was developed. To optimize the accuracy of the calibration, several mathematical pretreatments such as multiplicative scatter correction (MSC) and standard normal variate (SNV) were tested. MSC was used in order to remove optical interference and physical effects like particle size and surface blaze (Kapper et al., 2012a). SNV was tested as alternative to MSC in order to remove the multiplicative interferences of scatter and particle size (Barnes et al., 1989). Finally, SNV pre-treatment was not used in the models because the results obtained were worse than MSC pre-treatment.

To optimize the extraction of information from the spectra data, first or second derivatives were used to remove baseline offset variations (Savenije et al., 2006). Also, a Savitzky Golay filter was employed to improve the appearance of peaks obscured by random noise. Finally, outliers' spectra were identified and removed when necessary.
Evaluation of outliers and the mathematical pre-treatments were made using TQ Analyst 8.6.12 software (Thermo Fisher Scientific, Waltham, Massachusetts, USA).

\subsection{FT-NIRS calibration and chemometric analysis}

The total number of samples was split in two random data sets: a training set (calibration) comprising $80 \%$ of the samples, and a validation set including the remaining $20 \%$ of the samples. An internal full cross-validation using the leave one-out method was applied on the training set. Using the leave one-out method, the number of folds (the data subset portion) is equal to the number of instances in the full data set (Sammut and Webb, 2011).

Partial least squares (PLS) regression was applied to all models using TQ Analyst 8.6.12. For each parameter, the optimum number of factors used by PLS (FPLS) in the model development was the one that determined the lowest error in cross validation and thus avoiding overfitting (Prieto et al., 2014).

The best model for each trait was evaluated in terms of the highest coefficient of determination $\left(\mathrm{R}^{2}\right)$ and the lowest root-mean-square error (RMSE), in calibration ( $\mathrm{R}^{2} \mathrm{c}$; RMSEC), in cross-validation $\left(\mathrm{R}^{2} \mathrm{cv}\right.$; RMSECV) and in external validation ( $\mathrm{R}^{2} \mathrm{p}$; RMSEP), respectively. RMSE determines the performance of model regression and expresses the difference between the predicted and the measured references values (Lobos et al., 2013). Residual prediction deviation (RPD) was used in order to evaluate goodness of fit and model accuracy; it was calculated as the ratio between the standard deviation of the dataset divided by the root-mean-square error in calibration (RPDc $=\mathrm{SD} / \mathrm{RMSEC}$ ), in cross-validation (RPDcv $=$ SD/RMSECV) and in external validation $(\mathrm{RPDp}=\mathrm{SD} / \mathrm{RMSEP})$. The model performance can be considered sufficient for a rough screening if RPD is between 1.5 and 2.5 (Williams, 2014). Williams and Sobering (1993) suggested an 'accurate estimation capacity' if RPD values were higher than the limit of 2.5 , even though in the following years the limit for the accuracy evaluation was increased to 3 (Williams, 2014). Nevertheless, when reference data variance is low, the values for the $\mathrm{R}^{2}$ and the RPD cannot be very high (Pérez-Marín et al., 2004). The range error rate (RER) was calculated as the ratio between the range of sample values (reference techniques) and the RMSEP (Max-Min/RMSEP) (Pérez-Marín et al., 2004). RER values in the range of 4-8 suggest the possibility of discriminating between high and low values, while RER values in the range of 8-12 represent the possibility of predicting quantitative data (Barbin et al., 2015; Millmier et al., 2000). 


\section{Results and discussion}

\subsection{Descriptive statistics of meat quality traits}

A summary of descriptive statistics of the analysed meat quality traits is shown in Table 1. Lightness ( $\mathrm{L}^{*}$ ) was the only parameter that showed certain homogeneity. For the other three traits (yellowness b*, hardness and chewiness), the coefficient of variation was high (from $28.7 \%$ $37.0 \%$ ); and for the remaining traits the coefficient of variation was moderate (between $13.5 \%$ and $18.0 \%$ ).

The phenotypic data presented here showed similar values for CFL, $L^{*}, a^{*}$ and MB but slightly higher for $b^{*}$ when compared to animals from the same Iberian pig population presented earlier by Fernández-Barroso et al. (2020). These differences observed within the same population could be due to a strong environmental factor in the open-air free-range production system, where production traits of pigs reared in different seasons are strongly influenced by the weather, the harvest of acorns and the abundance of pastures. Moreover, some of the traits such as water content or fat deposition in muscle are inherently very heterogeneous in pork.

The comparison between the means and dispersion values of the meat quality traits analysed (Table 1) and those reported by other studies on the same traits in Iberian pigs (Estévez et al., 2003; Lopez-bote et al., 2008; Mayoral et al., 1999; Tejerina et al., 2012) is not straightforward due to the different experimental designs in terms of production system, slaughter age, muscle type or instrumental procedures. In addition, in our study, fresh meat samples were frozen, stored and then thawed for subsequent analyses (reference methods and NIRS) instead of performing the analysis on the same day that they were collected. The different freeze/thaw procedures probably affect the values obtained for the traits analysed. However, to carry out these analyses the same day that the samples were collected is unfeasible since an approximate number of 70 samples were collected for each slaughter date in a commercial slaughterhouse.

\subsection{NIRS spectra characteristics}

Average values of near infrared spectra, both for minced and intact samples from LTL muscle of Iberian pigs are shown in Fig. 2. The spectral information extracted from each type of sample showed a similar pattern, but intact meat seems to absorb slightly more energy than minced samples.

The differences in absorbance can be explained by the texture structure when meat is minced, which interferes with light absorbance, as suggested by Cozzolino et al. (2000) and Fan et al. (2018). Although several studies carried out with the NIRS methodology in other pig

\section{Table 1}

Descriptive statistics for traits from Longissimus thoracis et lumborum muscle of Iberian pigs fattened in an open-air free-range system.

\begin{tabular}{|c|c|c|c|c|c|c|}
\hline Trait & $\begin{array}{l}\text { Sample } \\
\text { size }\end{array}$ & Mean & SD & $\begin{array}{l}\text { CV } \\
(\%)\end{array}$ & Max & Min \\
\hline$L^{*}$ & 287 & 43.60 & 2.93 & 6.73 & 53.58 & 35.90 \\
\hline$a^{*}$ & 287 & 11.48 & 2.06 & 17.99 & 16.29 & 5.92 \\
\hline$b^{*}$ & 287 & 3.37 & 1.22 & 36.22 & 7.14 & 1.03 \\
\hline $\begin{array}{l}\text { Myoglobin ( } \mathrm{mg} \mathrm{g}^{-1} \\
\text { muscle) }\end{array}$ & 287 & 1.66 & 0.26 & 15.66 & 2.64 & 1.04 \\
\hline CFL (\%) & 287 & 29.98 & 4.04 & 13.47 & 37.49 & 16.52 \\
\hline Shear Force $\left(\mathrm{kg} \mathrm{cm}^{2}\right)$ & 287 & 4.39 & 0.73 & 16.68 & 6.61 & 1.96 \\
\hline Hardness $(\mathrm{N})$ & 287 & 9.34 & 2.68 & 28.69 & 20.31 & 2.68 \\
\hline Cohesiveness & 287 & 0.28 & 0.04 & 14.29 & 0.39 & 0.17 \\
\hline Springiness (mm) & 287 & 1.55 & 0.21 & 13.55 & 2.15 & 0.52 \\
\hline Chewiness ( $\mathrm{N}$ x mm) & 287 & 4.19 & 1.55 & 36.99 & 8.83 & 1.20 \\
\hline
\end{tabular}

Sample size: number of samples; SD: standard deviation; CV: coefficient of variation as a percentage; Max: maximum value; Min: minimum value; Minolta parameters: $\mathrm{L}^{*}$ lightness, $\mathrm{a}^{*}$ : redness, $\mathrm{b}^{*}$ : yellowness; CFL: centrifuge force water losses, TPA parameters: Hardness, cohesiveness, springiness, chewiness. breeds focused on evaluating some of the meat quality traits, such as instrumental colour and water holding capacity (Čandek-Potokar et al., 2006; Kapper et al., 2012b; Savenije et al., 2006; Wang et al., 2018), few references reported on the comparison between intact and minced samples. Cáceres-Nevado et al. (2019) also observed slightly higher absorbance of intact samples than minced ones when they analysed moisture, protein and fat content in Iberian pig loins. Ortiz-Somovilla et al. (2006) noted visual differences between different spectra types of pork products (minced and homogenized). Also, it seems that the homogenization of the products results in lower absorption due to the modification of the meat structure.

Furthermore, according to Cozzolino and Murray (2002), near infrared spectra in meat samples present high peaks of absorption (Fig. 2) that are linked to fat $\left(1190 \mathrm{~nm}\right.$ or $\left.8400 \mathrm{~cm}^{-1}\right)$, protein $(1550 \mathrm{~nm}$ or $6451 \mathrm{~cm}^{-1}$ ) and water $\left(1900 \mathrm{~nm}\right.$ or $\left.5260 \mathrm{~cm}^{-1}\right)$.

\subsection{NIRS prediction equations interpretation}

The summaries of the statistics obtained from calibration, crossvalidation and external validation models in minced and intact samples are showed in Tables 2 and 3, respectively. For every parameter, the optimal number of PLS factors used was included, in order to have a lower standard error, as well as the mathematical treatments. The wavelength is not specified because the full available near infrared region was used.

As expected, coefficients of determination obtained in calibration were higher than those of external validation models for both types of samples. In the minced samples (Table 2), most of the parameters ( $\mathrm{L}^{*}$, $\mathrm{MB}$, shear force, hardness, cohesiveness, springiness and chewiness) showed $R^{2} c$ values between 0.80 and 0.90 , while $R^{2} c$ values between 0.54 and 0.65 were lower for $\mathrm{a}^{*}, \mathrm{~b}^{*}$ and CFL. External validation reported lower $\mathrm{R}^{2} \mathrm{p}$ values than calibration models, ranging from 0.70 to 0.79 for $\mathrm{L}^{*}, \mathrm{MB}$, shear force, hardness, cohesiveness, springiness and chewiness and from 0.48 to 0.59 for $\mathrm{a}^{*}, \mathrm{~b}^{*}$ and CFL. In addition, traits with the highest $R^{2} c$ in calibration were also the highest for $R^{2} p\left(L^{*}, M B\right.$, shear force, hardness, cohesiveness, springiness and chewiness).

In the NIRS analyses carried out with intact samples (Table 3), the $\mathrm{R}^{2} \mathrm{c}$ values were higher than 0.80 only for two traits ( $\mathrm{b}^{*}$ and hardness) and ranged between 0.71 and 0.79 in $\mathrm{L}^{*}, \mathrm{a}^{*}, \mathrm{MB}, \mathrm{CFL}$, shear force, chewiness. Intermediate results were obtained in cohesiveness and springiness, with an $\mathrm{R}^{2} \mathrm{c}$ value equal to 0.68 . In the external validation, only hardness maintained a high $\mathrm{R}^{2} \mathrm{p}$ value of 0.80 , while the colour parameters $\mathrm{a}^{*}$ and $\mathrm{b}^{*}$ reached values of 0.70 and 0.73 , respectively. Lastly the remaining traits had an $\mathrm{R}^{2} \mathrm{p}$ value lower than 0.70 . Comparing the two sample presentation modes, $\mathrm{R}^{2}$ was higher in the intact than in the minced samples for four traits ( $a^{*}, b^{*} \mathrm{CFL}$ and hardness). The $\mathrm{R}^{2} \mathrm{cv}$ achieved the lowest results for all traits, both in minced and intact samples.

Regarding the residual prediction deviation (RPD), which takes into account the root-mean-square and the standard deviation of the trait, in minced samples shear force and CFL obtained RPDc values of 3.0 and 3.1 , respectively, while the values were equal to or greater than 1.5 in other traits. Only the colour parameters $\mathrm{a}^{*}$ and $\mathrm{b}^{*}$ showed RPDc values below 1.5. In the external validation, the RPDp values were lower than those of the calibration: only shear force and cohesiveness reached a value of 1.5 while the remaining traits showed values between 1.1 and 1.4 .

Again, in the analysis of intact samples (Table 3), the highest values of the statistics are clearly lower than in minced ones, since the maximum value of RPDc was 2.1 for hardness. However, RPDc values were equal to or higher than 1.5, for all the traits except for springiness. In validation, RPDp values ranged from 1.5 to 1.6 in $\mathrm{L}^{*}, \mathrm{a}^{*}, \mathrm{~b}^{*}$ and hardness, and between 1.0 and 1.4 in the remaining six traits. When comparing the RPD results of the two sample presentation modes, $\mathrm{L}^{*}, \mathrm{a}^{*}$, $\mathrm{b}^{*}$, CFL and hardness showed higher RPDp values in intact samples than in minced. 


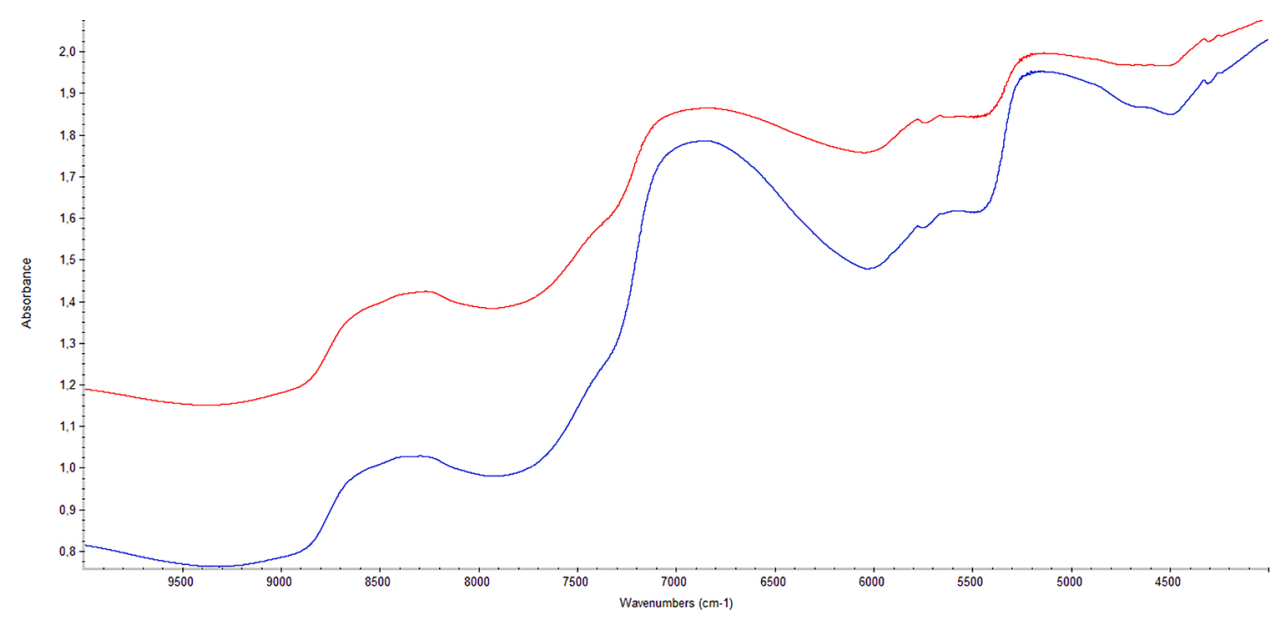

Fig. 2. Average NIR spectra of all minced (blue) and intact (red) Longissimus thoracis et lumborum samples of Iberian pigs fattened in an open-air free-range system (For interpretation of the references to colour in this figure legend, the reader is referred to the web version of this article).

Legend. The $\mathrm{Y}$-axis values correspond to the absorbance $(1 / \mathrm{R})$ of the samples along the different wavenumbers.

Table 2

Summary statistics for the PLS calibration and external validation models in minced samples.

\begin{tabular}{|c|c|c|c|c|c|c|c|c|c|c|c|c|c|c|}
\hline \multirow{2}{*}{ Trait } & \multirow{2}{*}{ Treatment } & \multirow{2}{*}{ FPLS } & \multicolumn{4}{|c|}{ Calibration } & \multirow{2}{*}{$\begin{array}{l}\text { Cross - } \\
\text { Validation } \\
\mathrm{R}^{2} \mathrm{cv}\end{array}$} & \multirow[b]{2}{*}{ RMSECV } & \multirow[b]{2}{*}{ RPDcv } & \multicolumn{5}{|c|}{ External validation } \\
\hline & & & $\mathrm{n}$ & $\mathrm{R}^{2} \mathrm{c}$ & RMSEC & RPDc & & & & $\mathrm{n}$ & $\mathrm{R}^{2} \mathrm{p}$ & RMSEP & RPDp & RER \\
\hline$L^{*}$ & $\begin{array}{l}\text { MSC + II deriv.+ SG } \\
\text { filter }\end{array}$ & 3 & 226 & 0.89 & 1.32 & 2.3 & 0.72 & 2.00 & 1.5 & 56 & 0.75 & 1.03 & 1.3 & 5.0 \\
\hline$a^{*}$ & $\begin{array}{l}\text { MSC + II deriv. + SG } \\
\text { filter }\end{array}$ & 3 & 217 & 0.54 & 1.73 & 1.2 & 0.48 & 1.32 & 1.6 & 54 & 0.48 & 1.07 & 1.1 & 4.6 \\
\hline$b^{*}$ & $\begin{array}{l}\text { MSC + II deriv. + SG } \\
\text { filter }\end{array}$ & 3 & 217 & 0.61 & 0.99 & 1.3 & 0.53 & 1.34 & 0.9 & 54 & 0.56 & 0.71 & 1.1 & 4.3 \\
\hline $\begin{array}{l}\text { Myoglobin (mg } \\
\mathrm{g}^{-1} \text { ) }\end{array}$ & MSC + II deriv. & 2 & 219 & 0.84 & 0.13 & 1.8 & 0.68 & 0.26 & 1.0 & 55 & 0.74 & 0.11 & 1.3 & 6.7 \\
\hline CFL (\%) & $\begin{array}{l}\text { MSC + II der. + SG } \\
\text { filter. }\end{array}$ & 2 & 216 & 0.65 & 1.39 & 3.0 & 0.49 & 2.80 & 1.5 & 53 & 0.59 & 1.37 & 1.3 & 4.4 \\
\hline $\begin{array}{l}\text { Shear Force }(\mathrm{kg} \\
\left.\mathrm{cm}^{2}\right)\end{array}$ & MSC + II deriv. & 2 & 218 & 0.80 & 0.23 & 3.1 & 0.66 & 0.77 & 0.9 & 55 & 0.70 & 0.44 & 1.9 & 10.6 \\
\hline Hardness (N) & MSC + II deriv. & 2 & 218 & 0.84 & 1.45 & 1.9 & 0.61 & 2.60 & 1.1 & 55 & 0.74 & 0.99 & 1.3 & 5.2 \\
\hline Cohesiveness & MSC + II deriv. & 3 & 222 & 0.90 & 0.02 & 2.4 & 0.68 & 0.02 & 1.6 & 56 & 0.79 & 0.02 & 1.5 & 6.1 \\
\hline Springiness (mm) & MSC + II deriv. & 2 & 205 & 0.86 & 0.11 & 2.0 & 0.69 & 0.17 & 1.3 & 51 & 0.79 & 0.08 & 1.4 & 6.1 \\
\hline $\begin{array}{l}\text { Chewiness ( } \mathrm{N} \mathrm{x} \\
\mathrm{mm} \text { ) }\end{array}$ & MSC + II deriv. & 2 & 214 & 0.87 & 0.85 & 2.0 & 0.65 & 1.25 & 1.4 & 53 & 0.78 & 0.50 & 1.3 & 4.6 \\
\hline
\end{tabular}

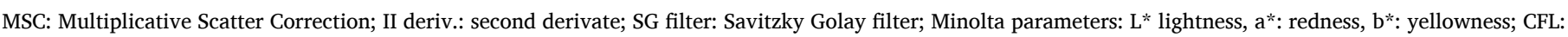

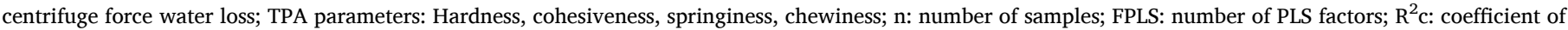

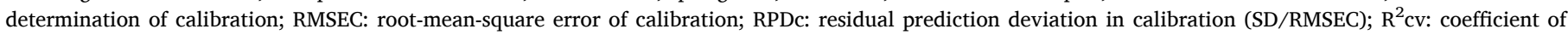

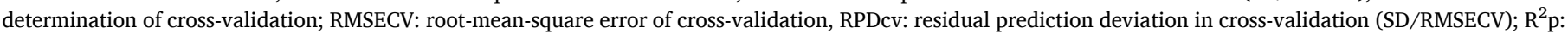

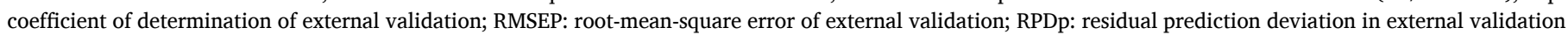
(SD/RMSEP); RER: range error ratio in external validation.

Finally, RER advises about the suitability to categorise or quantify the samples based on the spectra of the traits. All the traits showed RER values above 4 for the minced mode (Table 2). Even shear force reached a value equal to 10.6 , a result that implies a level of accuracy beyond classification and that would allow quantification with acceptable precision. Regarding the intact mode (Table 3), three traits had RER values below 4.0 (MB, cohesiveness and chewiness) and none exceeded 8.0.

Previous studies have reported a better performance of NIRS analyses in minced samples than in intact ones for moisture, protein, intramuscular fat content and fatty acid profile (Cáceres-Nevado et al., 2019; Fan et al., 2018; Ortiz et al., 2020) and for shear force (Barlocco et al., 2006). Nevertheless, to our knowledge, there are no studies that have measured myoglobin, CFL and TPA parameters in minced and intact samples. Intact muscles are heterogeneous samples with different chemical and physical characteristics due to different factors $(\mathrm{pH}$, myoglobin and protein precipitation in the sarcoplasm, fibre organization, myofibrillar birefringence, sarcomere length, moisture, intramuscular fat and macroscopic surface reflectance properties) (Cozzolino et al., 2000; Cozzolino and Murray, 2002). This heterogeneity could explain a worst performance of the NIRS analyses for some traits. However, in the present study, performances of the models were better in intact samples for $\mathrm{L}^{*}, \mathrm{a}^{*}, \mathrm{~b}^{*}, \mathrm{CFL}$ and hardness, since they showed higher RPDp and RER values than in minced samples (except a* for RER, which showed very similar values).

\subsection{NIRS evaluation by groups of traits}

\subsubsection{Colour parameters}

The prediction results of the MB by NIRS in intact samples were not very encouraging. RER was equal to 2.9 , advising against its implementation in the conditions of this experimental design. Better results were obtained in minced meat, although somewhat contradictory: while RER was equal to 6.7, within the range of discrimination between high and low values, the RPDp value was 1.3, below the recommended for a rough screening. To our knowledge, there is no study that calibrate NIRS for MB determination on porcine meat. Prieto et al. (2006) and Ripoll 
Table 3

Summary statistics for the PLS calibration and external validation models in intact samples.

\begin{tabular}{|c|c|c|c|c|c|c|c|c|c|c|c|c|c|c|}
\hline \multirow{2}{*}{ Trait } & \multirow{2}{*}{ Treatment } & \multirow{2}{*}{ FPLS } & \multicolumn{4}{|c|}{ Calibration } & \multicolumn{3}{|c|}{ Cross - Validation } & \multicolumn{5}{|c|}{ External validation } \\
\hline & & & $\mathrm{n}$ & $\mathrm{R}^{2} \mathrm{c}$ & RMSEC & RPDc & $\mathrm{R}^{2} \mathrm{cv}$ & RMSECV & RPDcv & $\mathrm{n}$ & $\mathrm{R}^{2} \mathrm{p}$ & RMSEP & RPDp & RER \\
\hline$L^{*}$ & MSC + I deriv. & 3 & 222 & 0.77 & 1.89 & 1.6 & 0.65 & 2.00 & 1.5 & 55 & 0.68 & 1.36 & 1.5 & 5.3 \\
\hline$a^{*}$ & MSC + I deriv. & 3 & 230 & 0.79 & 1.27 & 1.6 & 0.66 & 1.55 & 1.3 & 57 & 0.70 & 1.65 & 1.5 & 4.5 \\
\hline$b^{*}$ & MSC + I deriv. & 3 & 214 & 0.80 & 0.73 & 1.7 & 0.69 & 0.93 & 1.3 & 53 & 0.73 & 0.82 & 1.5 & 5.7 \\
\hline Myoglobin (mg g ${ }^{-1}$ ) & MSC + II deriv. & 3 & 225 & 0.71 & 0.15 & 1.7 & 0.61 & 0.31 & 0.8 & 56 & 0.67 & 0.18 & 1.2 & 2.9 \\
\hline CFL (\%) & MSC + II deriv. & 4 & 230 & 0.78 & 2.55 & 1.6 & 0.67 & 2.78 & 1.4 & 57 & 0.69 & 2.14 & 1.4 & 6.8 \\
\hline Shear Force $\left(\mathrm{kg} \mathrm{cm}^{2}\right)$ & MSC + II deriv. & 3 & 198 & 0.73 & 0.45 & 1.6 & 0.68 & 0.97 & 0.8 & 50 & 0.67 & 0.34 & 1.2 & 4.0 \\
\hline Hardness $(\mathrm{N})$ & MSC + II deriv. & 4 & 226 & 0.88 & 1.28 & 2.1 & 0.66 & 2.00 & 1.3 & 56 & 0.80 & 1.00 & 1.6 & 5.6 \\
\hline Cohesiveness & MSC + II deriv. & 3 & 195 & 0.68 & 0.03 & 1.5 & 0.53 & 0.04 & 0.9 & 49 & 0.61 & 0.03 & 1.0 & 3.4 \\
\hline Springiness (mm) & MSC + II deriv. & 4 & 207 & 0.68 & 0.16 & 1.3 & 0.58 & 0.24 & 0.9 & 52 & 0.60 & 0.17 & 1.2 & 5.5 \\
\hline Chewiness ( $\mathrm{N}$ mm) & MSC + II deriv. & 4 & 215 & 0.79 & 0.94 & 1.7 & 0.65 & 1.36 & 1.1 & 54 & 0.69 & 0.97 & 1.0 & 3.4 \\
\hline
\end{tabular}

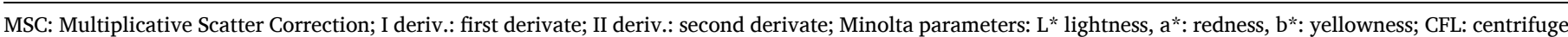

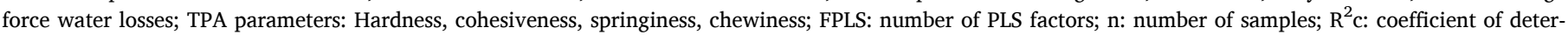

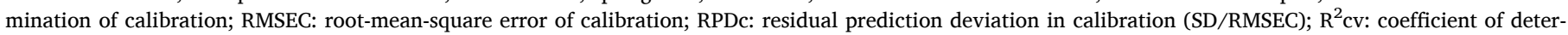

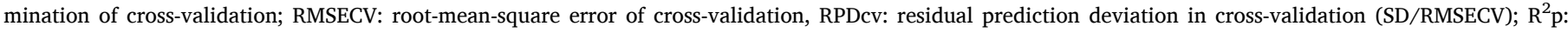

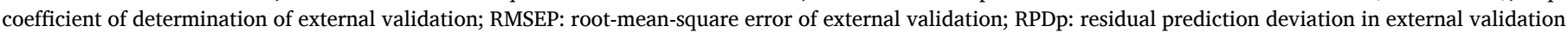
(SD/RMSEP); RER: range error ratio in external validation.

et al. (2008) reported RPD values of 1.09 and 2.38, respectively, in minced samples of cattle meat. The best results of Ripoll et al. (2008) were obtained working on the visible-near infrared spectrum range $(400-2500 \mathrm{~nm})$ and they observed a good MB estimation ability using the visible region $(400-750 \mathrm{~nm})$. The peak in the visible region approximately at $520 \mathrm{~nm}$ was probably related with the structure of meat pigments, such as myoglobin (Cozzolino et al., 2003; Cozzolino and Murray, 2002). Nevertheless, this wavenumber was not present in the near infrared region used in our study.

The observed results of RPDp in colour traits ( $L^{*}, a^{*}$ and $\left.b^{*}\right)$ were higher for intact (1.5) than for minced meat (1.1-1.3), which makes its implementation suitable directly on the raw material without prior homogenization. Several authors worked on the colour parameters and their prediction by NIRS. Candek-Potokar et al. (2006) observed slightly better results in the intact than in the minced mode in porcine Longissimus thoracis muscle using a similar wavelength to that used in the present study. Kapper et al. (2012b) reported better predicted statistics for $\mathrm{L}^{*}$ parameter than our findings, but worse for $\mathrm{a}^{*}$ and $\mathrm{b}^{*}$ on porcine intact LTL muscle. Monteiro-Balage et al. (2015) showed better accuracy than ours for the three colour parameters in intact porcine LTL. This higher accuracy may be linked to the region of the electromagnetic spectrum that they used: visible $(400-750 \mathrm{~nm})$ and a part of the near infrared region; in fact, Monteiro-Balage et al. (2015) showed that the selection of a specific set of waveband from 405 to $690 \mathrm{~nm}$ plays a relevant role improving the prediction accuracy of the colour parameters. Finally, Andrés et al. (2008) and Prieto et al. (2014) reported slightly better prediction ability for $L^{*}, a^{*}$ and $b^{*}$ in minced LTL samples of cattle trough the cross-validation procedure. This higher accuracy could be related to the electromagnetic spectrum used by Andrés et al. (2008) and Prieto et al. (2014) which also includes the region of the visible spectrum (400-2498 $\mathrm{nm})$.

\subsubsection{Centrifuge force loss and texture parameters}

Coefficients of determination in external validation for CFL in minced and intact samples were not high ( 0.59 and 0.69 , respectively) but RPDc was 3.0 in the minced mode, the second highest value of RPDc in the present study. However, the RPDp value decreased drastically in external validation (1.3), which was lower than the threshold necessary to categorise the samples. The best results in terms of RPDp and RER were achieved in intact mode, with a RER value of 6.8 . So far, no studies are available that report results for CFL in both porcine and cattle.

The prediction results observed for shear force (measured by WarnerBratzler) in minced samples were promising because RPD values (both in calibration and in external validation) and, above all, RER (equal to 10.6) would allow a quantification of this trait with moderate accuracy.
Previous studies on cooked samples of Longissimus thoracis from cattle reported similar results (Ripoll et al., 2008), while other studies reported poorer prediction estimates (Prieto et al., 2008; Andrés et al., 2008). These RPD and RER statistics were clearly lower in the intact than in minced mode. Nevertheless, our results presented higher predictive ability than those described for shear force by Barlocco et al. (2006); Cai et al. (2011) and Monteiro-Balage et al. (2015) in porcine cooked samples of Longissimus and by Bonin et al. (2020), in cooked intact samples of Longissimus thoracis from cattle.

Finally, regarding TPA parameters, our results presented better prediction equations in minced samples with the exception of hardness. However, results for hardness measured in minced samples presented better predictive ability than those reported by Ripoll et al. (2008). Even if the homogenisation of minced samples could have altered the muscle structure, our results suggest that this treatment did not have a great influence on rheological traits. Cohesiveness and springiness in minced samples presented RPDp values of 1.5 and 1.4, respectively, close to the threshold required to differentiate between low and high categories, with RER equal to 6.1 which allows samples to be categorised. Hardness in intact samples reached similar results: 1.6 and 5.6 for RPDp and RER, respectively. Chewiness was the TPA characteristic with the lowest prediction ability in both modes.

\subsection{NIRS predictive ability remarks}

The main objective of this work was to assess NIRS methodology to simultaneously measure a set of meat quality traits, as an alternative to instrumental measurements for further implementations such as in breeding programs or in industry quality control. In addition, two presentations of the sample were included, minced and intact, seeking to simplify the process by avoiding previous sample preparations and making it more accessible on a meat production line.

The general results in terms of $\mathrm{R}^{2}$, RMSE, RPD and RER reveals that NIRS cannot be used for an accurate and simultaneous quantification of the studied traits, as the accuracy required for strategies such as inclusion in breeding programs is not sufficient, but the results suggest the possibility of using NIRS to classify some of these traits into two or three categories. Shenk and Westerhaus (1996) set the range of $\mathrm{R}^{2}$ of prediction models from 0.50 to 0.69 for an adequate capacity to classify samples into three classes (high, medium, and low) and from 0.70 to 0.89 for a good predictive ability. All the $\mathrm{R}^{2} \mathrm{p}$ estimates shown in this work (except $\mathrm{a}^{*}$ in minced) are within the ranges indicated by these authors. RDPp values were equal to or greater than 1.5 in six traits: shear force, cohesiveness (in minced), $\mathrm{L}^{*}, \mathrm{a}^{*}, \mathrm{~b}^{*}$ and hardness (in intact); RER exceed 4.0 in all traits in minced samples and in seven traits in intact $\left(\mathrm{L}^{*}\right.$, 
$a^{*}, b^{*}, C F L$, shear force, hardness and springiness). These values are, in both statistics, the lowest value that are considered good enough for a rough screening (Williams, 2014) or to discriminate between high and low categories (Barbin et al., 2015; Millmier et al., 2000).

The results of the present study revealed that it is not possible to obtain a simultaneous quantification of the ten traits analysed with NIRS. From an individual point of view for each trait, although the predictive models allow a rough classification in intact samples of meat colour parameters $\left(L^{*}, a^{*}\right.$ and $\left.b^{*}\right)$, the measurement with the reference method using a colorimeter is even easier to implement than NIRS. The measurement of CFL in both cases (intact and minced) seems not to be accurate by NIRS, but considering that the usual analysis requires a long and laborious protocol, CFL could be indirectly measured quantifying the percentage of water losses by thawing and cooking due to their high genetic and phenotypic correlations (Fernández-Barroso et al., 2020).

Therefore, it is interesting to focus attention on $\mathrm{MB}$ and on texture (shear force and TPA parameters) which are not easy to quantify by traditional methods. MB is a very interesting quality trait for Iberian pigs (Ventanas et al., 2005) because consumers associate the bright cherry red colour with the high meat quality of animals raised in open-air free-range systems. However, its quantification process is long and laborious, especially when a large number of records are required. The NIRS prediction results shown here do not allow an accurate quantification of this trait. According to Cáceres-Nevado et al. (2019), the use of full spectra allows a large number of traits to be determined simultaneously. However, the region of the visible spectrum, which offers better results for MB (Prieto et al., 2006 and Ripoll et al., 2008), was not included in the considered spectral region.

Texture is also a remarkable quality trait of Iberian pigs, especially since the consumption of fresh meat has increased in the last decade. Shear force is one of the parameters related to texture, and to determinate it, a large number of replicates of the same sample have to be measured in the texturometer. Therefore, the use of NIRS instead of a texturometer would be clearly advantageous.

Tougher samples absorb more light than tender ones (Bonin et al., 2020) and this may be due to their shorter sarcomere length that allows light to penetrate easily (Andrés et al., 2007). This could explain the ability of NIRS to differentiate samples based on shear force. The results observed in this study, especially in minced samples, are very promising and encourage further research in order to improve the values of $\mathrm{R}^{2}$, RPD and RER. Simultaneous determination of shear force and TPA parameters using NIRS would be highly advantageous, since they could replace both complex sensory analysis through trained panellists and texturometer analyses.

According to Pérez-Marín et al. (2004), both an increase in the number of samples, and an increase in trait variation, as well as an improvement of the reference methods through a more rigid standardization could be relevant factors in improving the accuracy of the NIRS calibration model. Additionally, environmental conditions such as temperature or humidity, the length of the freezing period, and grinding or centrifuging processes should be meticulously controlled to reduce small cumulative laboratory errors, which could affect the precision of the estimation using NIRS. An improvement in the quantification made with the reference methods, reducing the variability between the replicates of an individual could be a relevant factor in improving the accuracy of the NIRS calibration model.

\section{Conclusion}

The results of this study revealed the difficulty of a single NIRS reading, using the full spectra range, to simultaneously quantify ten meat quality traits in samples of longissimus dorsi et lumborum from freerange Iberian pigs. However, NIRS seems to allow the quantification of shear force in minced samples, while a rough classification is possible both of cohesiveness in minced samples and of colour parameters L*, a*, $b^{*}$ and hardness in intact samples. Therefore, simplification of this methodology using intact rather than minced samples was not feasible for all traits.

The possibility of applying NIRS for shear force or hardness is relevant for meat quality control since they could be incorporated into the production line, just like other analyses already implemented (e.g., fatty acid profile or intramuscular fat content).

Further research is required to increase the number of samples and range of variation of the recorded traits, as well as to analyse different wavelengths for each individual trait or group of traits. The results observed for texture (shear force and TPA parameters) encourage new study designs to improve NIRS methodology for these meat quality traits, which are very relevant in the Iberian pig meat industry.

\section{Author contributions}

MA-FB participated in animal sampling, lab determinations, data analyses and drafting of the manuscript. JG-C obtained funding, participated in experimental design, animal sampling and review the manuscript. MM participated in experimental design, animal sampling and review the manuscript. P-PG contributed in lab determinations. GM and LR provided the animal material and participated in animal sampling. SP and RC contributed to NIRS calibration, bioinformatics analysis and review the manuscript. AC participated in data analyses.

\section{Declaration of Competing Interest}

All authors declare that they have not conflict of interest.

\section{Acknowledgments}

We want to thank to the company Sánchez Romero Carvajal (SRC) for the technical support. We want to thank to Wendy M. Rauw for her help revising the English style.

This work was financially supported by CON15-078, CON17-125 (INIA-SRC) and IDI- 20171141 (CDTI) grants. Miguel Ángel FernándezBarroso was funded by FPI Ph.D. grant from the INIA institution.

\section{References}

Alberti, P., Panea, B., Ripoll, G., Sañudo, C., Olleta, J.L., Hegueruela, I., Campo, M.M., Serra, X., 2005. Medición del color. In Estandarización de las metodologías para evaluar la calidad del producto (animal vivo, canal, carne y grasa) en los rumiantes. Serie Ganadería No 3. Instituto Nacional de Investigación y Tecnología Agraria y Alimentaria, Madrid (Spain), pp. 216-225.

Alves, E., Óvilo, C., Rodríguez, M.C. Silió, L., 2003, Mitochondrial DNA sequence variation and phylogenetic relationships among Iberian pigs and other domestic and wild pig populations. Anim. Genet. 34 (5), 319-324.

Andrés, S., Murray, I., Navajas, E.A., Fisher, A.V., Lambe, N.R., Bünger, L., 2007. Prediction of sensory characteristics of lamb meat samples by near infrared reflectance spectroscopy. Meat Sci. 76, 509-516.

Andrés, S., Silva, A., Soares-Pereira, A.L., Martins, C., Bruno-Soares, A.M., Murray, I., 2008. The use of visible and near infrared reflectance spectroscopy to predict beef M. longissimus thoracis et lumborum quality attributes. Meat Sci. 78 (3), 217-224.

Barbin, D.F., Kaminishikawahara, C.M., Lourenco Soares, A., Mizubuti, I.Y., Grespan, M., Shimokomaki, M., Hirook, E.Y., 2015. Prediction of chicken quality attributes by near infrared spectroscopy. Food Chem. 168, 554-560.

Barlocco, N., Vadell, A., Ballesteros, F., Galietta, G., Cozzolino, D., 2006. Predicting intramuscular fat, moisture and Warner-Bratzler shear force in pork muscle using near infrared reflectance spectroscopy. Anim. Sci. 82, 111-116.

Barnes, R.J., Dhanoa, M.S., Lister, S.J., 1989. Standard normal variate transformation and de-trending of near infrared diffuse reflectance spectra. Appl. Spectrosc. 43 (5), $772-777$.

Bonin, M., da Luz e Silva, S., Bünger, L., Ross, D., Dias Feijó, G.L., da Costa Gomes, R., Palma Rennó, F., de Almeida Santana, M., Marcondes de Rezende, F., Vinhas Ítavo, L.C., de Novais, F.J., Antonia Surita, L.M., Filgueira Pereira, M.W., Ferraz, J.B. S., 2020. Predicting the shear value and intramuscular fat in meat from Nellore cattle using Vis-NIR spectroscopy. Meat Sci. 163, 108077.

Bourne, M.C., 1978. Texture profile analysis. Food Technol. 32 (62-66), 72.

Cáceres-Nevado, J.M., Garrido-Varo, A., De Pedro-Sanz, E., Pérez-Marín, D.C., 2019. Fourier transform near-infrared spectroscopy coupled to a long fibre optic head for the quality control of IBERIAN pork loins: Intact versus minced. Meat Sci. 153, 86-93. https://doi.org/10.1016/j.meatsci.2019.03.008. 
Cai, J., Chen, Q., Wan, X., Zhao, J., 2011. Determination of total volatile basic nitrogen (TVB-N) content and Warner-Bratzler shear force (WBSF) in pork using Fourier transform near infrared (FT-NIR) spectroscopy. Food Chem. 126 (3), 1354-1360.

Čandek-Potokar, M., Prevolnik, M., Skrlep, M., 2006. Ability of near infrared spectroscopy to predict pork technological traits. J. Near Infrared Spectrosc. 14 (4), 269-277.

Cozzolino, D., Murray, I., 2002. Effect of sample presentation and animal muscle species on the analysis of meat by near infrared reflectance spectroscopy. J. Near Infrared Spectrosc. 10, 37-44.

Cozzolino, D., Murray, I., Scaife, J.R., Paterson, R., 2000. Study of dissected lamb muscles by visible and near infrared reflectance spectroscopy for composition assessment. Anim. Sci. 70, 417-423.

Cozzolino, D., Barlocco, N., Vadell, A., Ballesteros, F., Gallieta, G., 2003. The use of visible and near-infrared reflectance spectroscopy to predict colour on both intact and homogenised pork muscle. LWT - Food Sci. Technol. 36, 195-202.

Estévez, M., Morcuende, D., Cava López, R., 2003. Physico-chemical characteristics of M. Longissimus dorsi from three lines of free-range reared Iberian pigs slaughtered at 90 kg live-weight and commercial pigs: a comparative study. Meat Sci. 64 (4), 499-506.

Fan, Y., Liao, Y., Cheng, F., 2018. Predicting of intramuscular fat content in pork using near infrared spectroscopy and multivariate analysis. Int. J. Food Prop. 21 (1), 1180-1189.

Fernández-Barroso, M.Á., Silió, L., Rodríguez, C., Palma-granados, P., López, A., Caraballo, C., García-Casco, J.M., Sánchez-Esquiliche, F., Gómez-Carballar, F., Muñoz, M., 2020. Genetic parameter estimation and gene association analyses for meat quality traits in open-air free-range Iberian pigs. J. Anim. Breed. Genet. 1-18. https://doi.org/10.1111/jbg.12498.

Honikel, K., 1998. Reference methods for the assessment of physical characteristics of meat. Meat Sci. 49 (4), 447-457.

Hornsey, H.C., 1956. The colour of cooked cured pork. 1. Estimation of the nitric oxide-Haem pigments. J. Sci. Food Agric. 7 (8), 534-540.

Kapper, C., Klont, R.E., Verdonk, J., Urlings, H.A.P., 2012a. Prediction of pork quality with near infrared spectroscopy (NIRS) 2. Feasibility and robustness of NIRS measurements under production plant conditions. Meat Sci. 91 (3), 300-305.

Kapper, C., Klont, R.E., Verdonk, J., Urlings, H.A.P., 2012b. Prediction of pork quality with near infrared spectroscopy (NIRS): 1 Feasibility and robustness of NIRS measurements at laboratory scale. Meat Sci. 91 (3), 294-299.

Lobos, I., Gou, P., Hube, S., Saldana, R., Alfaro, M., 2013. Evaluation of potential NIRS to predict pastures nutritive value. J. Soil Sci. Plant Nutr. 13, 463-468.

López-Bote, C.J., 1998. Sustained utilization of the Iberian pig breed. Meat Sci. 49 $17-27$.

Lopez-Bote, C.J., Toldrá, F., Daza, A., Ferrer, J.M., Menoyo, D., Silió, L., Rodríguez, M.C., 2008. Effect of exercise on skeletal muscle proteolytic enzyme activity and meat quality characteristics in Iberian pigs. Meat Sci. 79 (1), 71-76.

Mayoral, A.I., Dorado, M., Guillen, M.T., Robina, A., Vivo, J.M., Vazquez, C., Ruiz, J., 1999. Development of meat and carcass quality characteristics in Iberian pigs reared outdoors. Meat Sci. 52, 315-324.

Millmier, A., Lorimor, J., Hurburgh, C., Fulhage, C., Hattey, J., Zhang, H., 2000. Nearinfrared sensing of manure nutrients. Trans. ASAE 43 (4), 903-908.

Monteiro-Balage, J., da Luz, S., Abdalla Gomide, C., de Nadai Bonin, M., Figueira, A.C., 2015. Predicting pork quality using Vis/NIR spectroscopy. Meat Sci. 108, 37-43.

Ollivier, L., 2009. European pig genetic diversity: a minireview. Animal 3 (07), 915-924.

Ortiz, A., Parrini, S., Tejerina, D., Pinto de Araújo, J.P., Čandek-Potokar, M., Crovetti, A., Garcia-Casco, J.M., González, J., Hernández-García, F.I., Karolyi, D., Margeta, V., Martins, J.M., Nieto, R., Petig, M., Razmaite, V., Sirtori, F., Lebret, B., Bozzi, R., 2020. Potential use of near-infrared spectroscopy to predict fatty acid profile of meat from different European autochthonous pig breeds. Appl. Sci. 10, 5801.

Ortiz-Somovilla, V., Espana-Espana, F., Gaitan-Jurado, A.J., Perez-Aparicio, J., De PedroSanz, E.J., 2006. Proximate analysis of homogenized and minced mass of pork sausages by NIRS. Food Chem. 101 (3), 1031-1040.

Osborne, B.G., 2000. Near-infrared spectroscopy in food analysis. Encyclopedia of Analytical Chemistry, pp. 1-14. https://doi.org/10.1002/9780470027318.a1018.

Parrini, S., Acciaioli, A., Crovetti, A., Bozzi, R., 2017. Use of FT-NIRS for determination of chemical components and nutritional value of natural pasture. Ital. J. Anim. Sci. 17 (1), 87-91.

Pérez-Marín, D.C., Garrido-Varo, A., Guerrero-Ginel, J.E., Gómez-Cabrera, A., 2004. Near-infrared reflectance spectroscopy (NIRS) for the mandatory labelling of compound feeding stuffs: chemical composition and open-declaration. Anim. Feed Sci. Technol. 116 (3-4), 333-349.

Pérez-Marín, D., De Pedro, E., Guerrero-Ginel, J.E., Garrido-Varo, A., 2009. A feasibility study on the use of near-infrared spectroscopy for prediction of the fatty acid profile in live Iberian pigs and carcasses. Meat Sci. 83, 627-633.

Prieto, N., Andrés, S., Giráldez, F.J., Mantecón, A.R., Lavín, P., 2006. Potential use of near infrared reflectance spectroscopy (NIRS) for the estimation of chemical composition of oxen meat samples. Meat Sci. 74 (3), 487-496.

Prieto, N., Andrés, S., Giráldez, F.J., Mantecón, A.R., Lavín, P., 2008. Ability of near infrared reflectance spectroscopy (NIRS) to estimate physical parameters of adult steers (oxen) and young cattle meat samples. Meat Sci. 79 (4), 692-699.

Prieto, N., Roehe, R., Lavín, P., Batten, G., Andrés, S., 2009. Application of near infrared reflectance spectroscopy to predict meat and meat products quality: a review. Meat Sci. 83, 175-186.

Prieto, N., López-Campos, Ó., Aalhus, J.L., Dugan, M.E.R., Juárez, M., Uttaro, B., 2014. Use of near infrared spectroscopy for estimating meat chemical composition, quality traits and fatty acid content from cattle fed sunflower or flaxseed. Meat Sci. 98 (2), 279-288.

Prieto, N., Juárez, M., Larsen, I.L., López-Campos, Ó., Zijlstra, R.T., Aalhus, J.L., 2015. Rapid discrimination of enhanced quality pork by visible and near infrared spectroscopy. Meat Sci. 110, 76-84.

Ripoll, G., Albertí, P., Panea, B., Olleta, J.L., Sañudo, C., 2008. Near-infrared reflectance spectroscopy for predicting chemical, instrumental and sensory quality of beef. Meat Sci. 80 (3), 697-702.

Sammut, C., Webb, G.I., 2011. Leave-one-out cross-validation. Encyclopedia of Machine Learning. Springer, Boston, MA. https://doi.org/10.1007/978-0-387-30164-8 469.

SanCristobal, M., Chevalet, C., Haley, C.S., Joosten, R., Rattink, A.P., Harlizius, B., Groenen, M.A., Amigues, Y., Boscher, M.Y., Russell, G., Law, A., Davoli, R., Russo, V., Désautés, C., Alderson, L., Fimland, E., Bagga, M., Delgado, J.V., VegaPla, J.L., Martinez, A.M., Ramos, M., Glodek, P., Meyer, J.N., Gandini, G.C., Matassino, D., Plastow, G.S., Siggens, K.W., Laval, G., Archibald, A.L., Milan, D., Hammond, K., Cardellino, R., 2006. Genetic diversity within and between European pig breeds using microsatellite markers. Anim. Genet. 37 (3), 189-198.

Savenije, B., Geesink, G.H., Van Der Palen, J.G.P., Hemke, G., Hopkins, D., Ouali, A., 2006. Prediction of pork quality using visible/near-infrared reflectance spectroscopy. Meat Sci. 73 (1), 181-184.

Shenk, J., Westerhaus, M., 1996. Calibration the ISI Way. In: Davis, A.M.C., Williams, P. (Eds.), Near Infrared Spectroscopy: The Future Waves, NIR Publications, pp. 198-202. Chichester (U.K.).

Solís, M., De Pedro, E., Garrido, A., García, J., Silió, L., Rodríguez, C., Rodrigañez, J., 2001. Evaluación de la composición del lomo de cerdo ibérico mediante la tecnología NIRS. In: Zaragoza (Spain)Proceedings of the Asociación Interprofesional para el Desarrollo Agrario - ITEA, II, pp. 613-615.

Tejerina, D., García-Torres, S., Cava, R., 2012. Water-Holding capacity and instrumental texture properties of M. Longissimus Dorsi and M. Serratus Ventralis from Iberian Pigs as affected by the production system. Livest. Sci. 148 (1-2), 46-51.

Tejerina, D., García-Torres, S., Cabeza de Vaca, M., Ortiz, A., Romero-Fernández, M.P., 2018. Evaluation of near-infrared spectroscopy (NIRS) for the quality control of packaged cured ham-sliced from Iberian pigs. Archivos de Zootecnia. Proceedings IX Simposio Internacional sobre el Cerdo Mediterráneo 231-234. https://doi.org/ 10.21071/az.v67iSupplement.3611.

Ventanas, S., Ventanas, J., Ruiz, J., Estévez, M., 2005. Iberian pigs for the development of high-quality cured products. Proc. Recent Res. Develop. Agric. Food Chem. 6 (27-53). Research Signpost, Kerala, (India).

Wang, W., Peng, Y., Sun, H., Zheng, X., Wei, W., 2018. Real-time inspection of pork quality attributes using dual-band spectroscopy. J. Food Eng. 237, 103-109.

Williams, P., 2014. The RPD statistic: a tutorial note. NIR News 25, 22-26.

Williams, P.C., Sobering, D.C., 1993. Comparison of commercial near infrared transmittance and reflectance instruments for analysis of whole grains and seeds. J. Near Infrared Spectrosc. 1, 25-32.

Zamora-Rojas, E., Pérez-Marín, D., De Pedro-Sanz, E., Guerrero-Ginel, J.E., GarridoVaro, A., 2012. In-situ Iberian pig carcass classification using a micro-electromechanical system (MEMS)-based near infrared (NIR) spectrometer. Meat Sci. 90, 636-642.

Zamora-Rojas, E., Garrido-Varo, A., De Pedro-Sanz, E., Guerrero-Ginel, J.E., PérezMarín, D., 2013. Prediction of fatty acid content in pig adipose tissue by near infrared spectroscopy: at-line versus in-situ analysis. Meat Sci. 95 (3), 503-511. 